\title{
Health-related quality of life in esophageal cancer: Effect of neoadjuvant chemoradiotherapy followed by surgical intervention
}

\author{
Najib Safieddine, MD, ${ }^{a}$ Wei Xu, PhD, ${ }^{\text {b }}$ Sayed Mohammed Quadri, MD, FRCSC, ${ }^{c}$ Jennifer J. Knox, MD, FRCP, ${ }^{\mathrm{d}}$ \\ Jennifer Hornby, BSc, CCRP, ${ }^{\mathrm{a}}$ Joanne Sulman, MSW, ${ }^{\mathrm{e}}$ Rebecca Wong, MD, FRCP, ${ }^{\mathrm{f}}$ Maha Guindi, MD, FRCP, ${ }^{\mathrm{g}}$ \\ Shaf Keshavjee, MD, MSc, FRCSC, FACS, ${ }^{a}$ and Gail Darling, MD, FRCSC, FACS ${ }^{\mathrm{a}}$
}

Objective: We sought to determine the effect of neoadjuvant chemoradiotherapy followed by surgical intervention on health-related quality of life in patients with esophageal cancer.

\begin{abstract}
Methods: Health-related quality of life was evaluated in a prospective phase II study of neoadjuvant chemoradiotherapy followed by esophagectomy in 52 patients with carcinoma of the esophagus. Esophagectomy was performed 6 weeks after completion of induction. Functional Assessment of Cancer Therapy-Esophageal scoring was performed before treatment, 7 weeks after initiation of neoadjuvant therapy, before resection, and at 1, 3, and 6 months and 1 year after resection.
\end{abstract}

Results: Forty-three patients completed the entire treatment protocol. Functional Assessment of Cancer TherapyEsophageal scores decreased significantly after chemoradiation at week 7 (120 vs 127 at baseline, $P=.04)$ but returned to baseline levels before surgical intervention (127). Similarly, scores decreased significantly after surgical intervention (115 at 1 month, $P=.02$ ) but returned to baseline levels by 3 months postoperatively (127). At 1 year postoperatively, there was a statistically significant improvement in scores compared with those at baseline $(139, P=.003)$. Functional Assessment of Cancer Therapy-Esophageal scores continued to increase over time for patients who were alive at least 1 year after the operation with or without disease but were observed to significantly decrease in those who died within 1 year after the operation $(P=.0001)$. An increase in quality of life was associated with a significantly lower risk of death $(P=.04)$.

Conclusion: Neoadjuvant therapy has a significant effect on health-related quality of life, but this is transient, with recovery to baseline within 5 to 7 weeks after completion of induction therapy. Health-related quality of life decreases again after surgical intervention but returns to baseline levels within 3 months.

Earn CME credits at

http://cme.ctsnetjournals.org

The primary objective of this study was to assess the effect of neoadjuvant chemoradiation followed by surgical intervention for esophageal cancer on health-related quality of life (HRQOL) by using the Functional Assessment of Can-

\footnotetext{
From the Division of Thoracic Surgery, ${ }^{a}$ Department of Surgery, Toronto General Hospital, University of Toronto, Toronto, Ontario, Canada; the Department of Biostatistics, ${ }^{\text {b }}$ Public Health Sciences, University of Toronto, Toronto, Ontario, Canada; the Division of Thoracic Surgery, ${ }^{\mathrm{c}}$ University of British Columbia, Vancouver, British Columbia, Canada; the Department of Medicine, ${ }^{\mathrm{d}}$ Princess Margaret Hospital, University of Toronto, Toronto, Ontario, Canada; the Department of Social Work, ${ }^{\mathrm{e}}$ Mount Sinai Hospital, University of Toronto, Toronto, Ontario, Canada; the Department of Radiation Oncology ${ }^{\mathrm{f}}$ Princess Margaret Hospital, University of Toronto, Toronto, Ontario, Canada; and the Department of Pathology, ${ }^{\mathrm{g}}$ Toronto General Hospital, University of Toronto, Toronto, Ontario, Canada.

Read at the Eighty-seventh Annual Meeting of The American Association for Thoracic Surgery, Washington, DC, May 5-9, 2007.

Received for publication March 29, 2007; revisions received Sept 24, 2008; accepted for publication Sept 30, 2008

Address for reprints: Gail Darling, MD, FRCSC, FACS, Toronto General Hospital, Division of Thoracic Surgery, 9N-955, 200 Elizabeth St, Toronto, Ontario M5G 2C4, Canada (E-mail: gail.darling@uhn.on.ca).

J Thorac Cardiovasc Surg 2009;137:36-42

$0022-5223 / \$ 36.00$

Copyright (c) 2009 by The American Association for Thoracic Surgery doi:10.1016/j.jtcvs.2008.09.049
}

cer Therapy-Esophageal (FACT-E). FACT-E is a validated tool to measure the effect of treatment on functional, social, physical, and emotional well-being that incorporates the esophageal cancer subscale and allows for a systematic evaluation of quality of life (QOL) specifically in the context of esophageal cancer. ${ }^{1}$ A prospective cohort of 52 patients with esophageal cancer who underwent neoadjuvant chemoradiation therapy followed by surgical resection was studied.

With an increasing incidence and an overall survival of $5 \%$ to $8 \%$, esophageal cancer is one of the most lethal malignancies. Although surgical intervention remains the mainstay of treatment for resectable disease, with a 5-year survival rate of $25 \%$ to $40 \%,{ }^{2-8}$ meta-analyses of neoadjuvant therapy followed by surgical intervention have suggested a survival benefit, albeit a modest one at best. ${ }^{9}$ Also, neoadjuvant therapy might improve resectability in locally advanced disease because of a downstaging effect. ${ }^{10}$ However, the morbidity associated with neoadjuvant chemoradiation is significant and entails a prolonged period of treatment (3 months), which represents almost $20 \%$ of the historical median survival time of 16.3 months. ${ }^{11}$

This paragraph should be rewritten: The effect of neoadjuvant treatment on QOL assumes greater significance in a disease with a poor prognosis, significant treatment related morbidity and only modest survival benefit. Measurement of 


\author{
Abbreviations and Acronyms \\ EORTC $=$ European Organization for \\ Research and Treatment and Cancer \\ FACT-E $=$ Functional Assessment of Cancer \\ Therapy-Esophageal \\ HRQOL $=$ health-related quality of life \\ QOL $=$ quality of life \\ SF-36 = Short Form-36
}

\section{RESULTS}

From November 2002 to October 2005, 215 patients with esophageal cancer presented to our institution, of which 102 were considered operative candidates. Of the operative candidates, 68 patients were evaluated for inclusion in the trial, and 53 were eligible. Only 1 patient declined. The mean age was 60 years (range, $33-79$ ), and $77 \%$ were men. The tumor was located at the gastroesophageal junction in $15(29 \%)$ patients and in the thoracic esophagus in $37(71 \%)$ patients. Histology was adenocarcinoma in $37(71 \%)$ patients, squamous cell carcinoma in $13(25 \%)$ patients, and poorly differentiated large cell carcinoma in $2(4 \%)$ patients. Based on clinical staging, 12 patients had T2 disease, 35 has T3 disease, and 5 had T4 disease; 15 patients had N0 disease and 37 had N1 disease; and 41 patients had M0 disease and 11 had M1A disease. The clinical stage at enrollment was IIA in $13(25 \%)$ patients, IIB in $8(15 \%)$ patients, III in 20 $(39 \%)$ patients, and IVA in $11(21 \%)$ patients.

Of the 52 enrolled patients, 3 discontinued induction therapy because of toxicity and proceeded directly to surgical intervention, and 2 refused further treatment during preoperative chemoradiotherapy. There were 2 preoperative deaths caused by stroke and central line sepsis. Preoperative treatment was completed in 45 patients, but 2 progressed on and were not treated surgically; the remaining 43 proceeded to surgical intervention. The median time between the completion of neoadjuvant treatment and surgical resection was 6.86 weeks (range, 5-11 weeks) (Fig 1). Thirty-day mortality was $2 \%$ ( 1 patient), and 2 other patients died in the hospital at 51 and 95 days postoperatively (death caused by pneumonia and recurrent cancer, respectively). Another 7 patients died within 1 year of surgical intervention, all because of recurrent cancer. Pathologic response data are available on 43 patients and have been previously reported. ${ }^{12}$

The mean, median, minimum, and maximum FACT-E scores for all patients who completed the treatment are listed in Table 1 (scores are missing for some time points, and all available data are reported). FACT-E scores decreased significantly after chemoradiation at week 7 (120 vs 127 baseline, $P=.04)$ but returned to baseline before surgical intervention (127). Similarly, scores decreased significantly after surgical

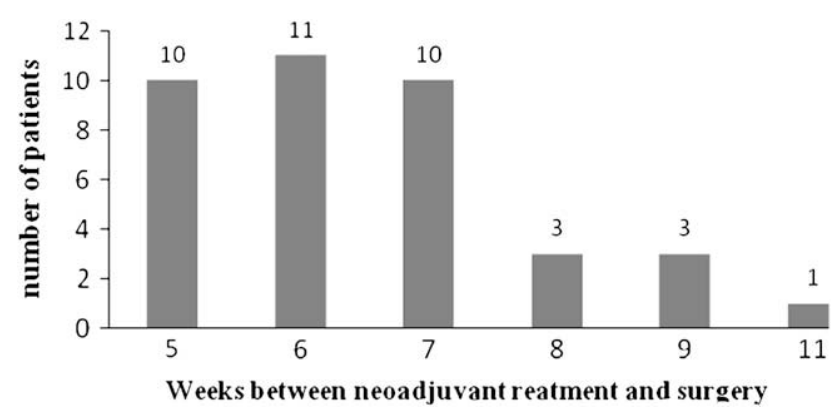

FIGURE 1. Number of patients versus waiting time between neoadjuvant treatment and surgical intervention. with a left neck incision for the anastomosis. En bloc resection was performed, including the overlying mediastinal pleura and periesophageal tissues with $5-\mathrm{cm}$ proximal and distal margins. Lymphadenectomy was performed, including the mid and lower mediastinal nodes and a D2 dissection in the abdomen. Transhiatal esophagectomy was allowed but not recommended.

This study and the use of all data collected were approved by the research and ethics board at our institution. Demographic factors and clinical and pathologic stage were summarized by using median and range (for continuous variables) and frequencies (for categorical variables). The paired $t$ test was applied to compare the FACT-E scores at different time points. Mixedmodel regression was applied to model the repeated measurement of FACTE scores over time and estimate the random intercept and slope of the FACT-E score. The estimated random slopes were used in the Cox proportional hazard model as predictors for the patients' overall survival. The statistical analysis was performed with version 9.1 of the SAS System and User's Guide (SAS Institute, Inc, Cary, NC). 
TABLE 1. FACT-E scores

\begin{tabular}{lccccccc}
\hline & No. & Mean & Median & Standard deviation & Standard error* & Minimum & Maximum \\
\hline Pre-TX & 40 & 126.8 & 127.0 & 24.0 & 7.34 & 60.3 & 167.0 \\
7 weeks postTx & 38 & 119.9 & 125.0 & 26.1 & 7.36 & 7.38 & 165.0 \\
PreSx & 38 & 127.4 & 137.7 & 28.0 & 7.41 & 45.8 \\
1 mo postop & 33 & 115.3 & 114.0 & 21.0 & 7.44 & 78.0 \\
3 mo postop & 33 & 126.7 & 129.5 & 24.8 & 7.49 & 66.0 \\
1 y postop & 28 & 138.6 & 145.8 & 22.1 & 96.0 & 156.0 \\
\hline
\end{tabular}

FACT-E, Functional Assessment of Cancer Therapy-Esophageal; PreTx, Before treatment; PostTx, after treatment; PreSx, before resection; postop, postoperatively.

intervention (115 at 1 month, $P=.02$ ) but returned to baseline by 3 months postoperatively (127). Changes in FACT-E scores are shown in Figures 2 through 4 . At 1 year postoperatively, there was a statistically significant improvement in scores compared with those seen at baseline (127 vs 139 , $P=.003)$. FACT-E scores continued to increase over time for patients who were alive at least 1 year after the operation with or without disease but were observed to significantly decrease in those who died within 1 year after surgical intervention $(P=.0001)$. An increase in QOL score was associated with a significantly lower risk of death $(P=.04)$.

In analyzing the subscales of the FACT-E, there was a significant decrease in scores for physical and functional wellbeing after neoadjuvant chemotherapy (Figures 5 and 6), whereas emotional and social well-being scores remained fairly stable.

The esophageal cancer-specific concerns reflected in the esophageal cancer subscale improved with neoadjuvant chemoradiation and then decreased after surgical intervention but recovered by 3 months and then increased over time (Figure 7).

Importantly, dysphagia was improved or relieved with preoperative treatment in two thirds of patients in whom it was a presenting complaint (data not shown). Only 6 patients required feeding tubes in this trial, 2 inserted before the start of treatment because of poor nutritional status and 4 inserted during chemoradiation.

Similarly, eating and swallowing indices ${ }^{12}$ increased significantly with neoadjuvant therapy. After surgical intervention, there was a decrease in both scores but only minimally

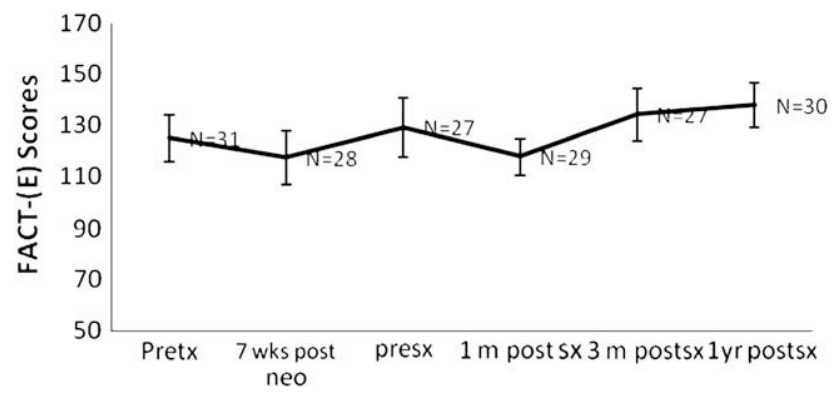

FIGURE 2. Mean Functional Assessment of Cancer Therapy-Esophageal (FACT-[E]) scores versus time: patients alive at 1 year $(\mathrm{n}=33)$. Pretx, Before treatment; neo, 7 weeks after initiation of neoadjuvant therapy; presx, before resection; postsx, after resection. for swallowing, whereas the eating score decreased significantly. By 3 months postoperatively, the swallowing index was back to preoperative levels and then continued to improve. The eating index was slower to recover but steadily increased over the ensuing 24 months that these patients were followed.

The induction regimen was well tolerated, with low rates of grade 3 or 4 anorexia (14 patients), fatigue (12 patients), nausea/vomiting (10 patients), and diarrhea (10 patients). Febrile neutropenia occurred in 8 patients, with grade 4 neutropenia in 13 patients.

\section{DISCUSSION}

Although combined modality therapy (chemotherapy/ radiation therapy/surgical intervention) is arduous and prolonged, its effect on HRQOL in patients with operable esophageal cancer is transient as because HRQOL scores return to baseline levels after induction and before surgical intervention. Similarly, surgical intervention has a significant effect on HRQOL because FACT-E scores decrease significantly 1 month after surgical intervention but again return to baseline levels within 3 months of surgical intervention. Significantly greater increases in FACT-E scores were observed in patients who were still alive 1 year after surgical intervention with or without disease but were observed to decrease in those who died within 1 year of surgical intervention.

Our findings are in contrast to previous work by Blazeby and colleagues using the European Organization for

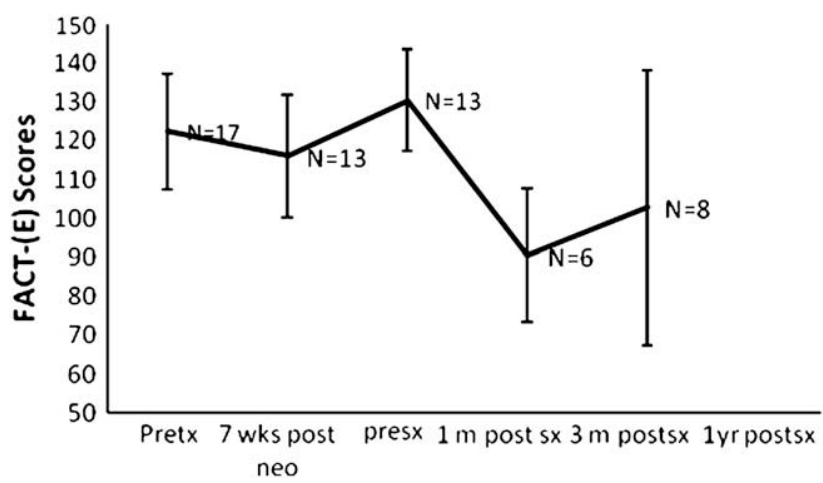

FIGURE 3. Mean Functional Assessment of Cancer Therapy-Esophageal (FACT-[E]) scores versus time: died before 1 year $(\mathrm{n}=17)$. Pretx, Before treatment; neo, 7 weeks after initiation of neoadjuvant therapy; pres $x$, before resection; postsx, after resection. 


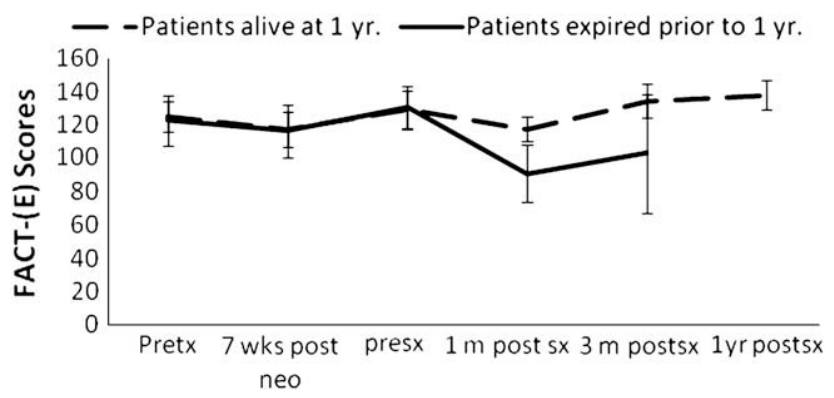

FIGURE 4. Mean Functional Assessment of Cancer Therapy-Esophageal (FACT-[E]) scores versus time $(\mathrm{n}=50)$. Pretx, Before treatment; neo, 7 weeks after initiation of neoadjuvant therapy; presx, before resection; postsx, after resection.

Research and Treatment and Cancer (EORTC)-QLQ 30 and OES 18, who reported that patients 3 to 4 months after esophagectomy have poorer QOL scores compared with that at baseline. ${ }^{13,14}$ Others have reported that patients after esophagectomy have a poorer QOL score compared with the normal population, which is not entirely unexpected. ${ }^{15}$ It is not clear why the patients in our study reported QOL scores similar to baseline values 3 months after esophagectomy. We had similar findings in patients after esophagectomy who had not been treated with induction chemoradiotherapy. This does not seem to be related to the QOL instrument used, because Brooks and associates, ${ }^{16}$ using the FACT-E, also reported reduced QOL scores 3 months after esophagectomy and found that QOL scores did not return to baseline until 9 months after esophagectomy. They also reported that the patients who received induction chemoradiation did not regain baseline HRQOL scores, even beyond 9 months. Although not detailed, most likely 5-fluoruracil-cisplatin-based therapy was used because that was the standard practice at the time of their study. It was our impression that the combination of irinotecan and cisplatin seemed to be associated with less dysphagia and esophagitis compared with 5 fluorouracil-cisplatin and that the regimen used in our study was well tolerated, with almost no need for nutritional support and low rates of fatigue, anorexia, diarrhea, and nausea.

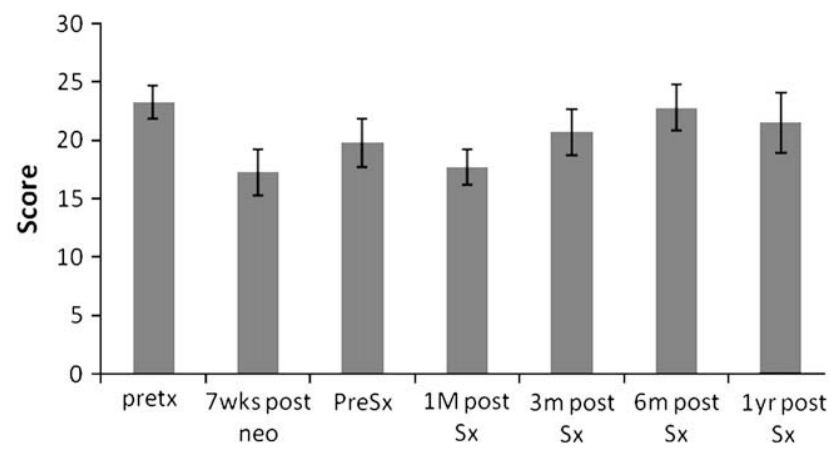

FIGURE 5. Physical well-being scores. Pretx, Before treatment; neo, 7 weeks after initiation of neoadjuvant therapy; presx, before resection; postsx, after resection.

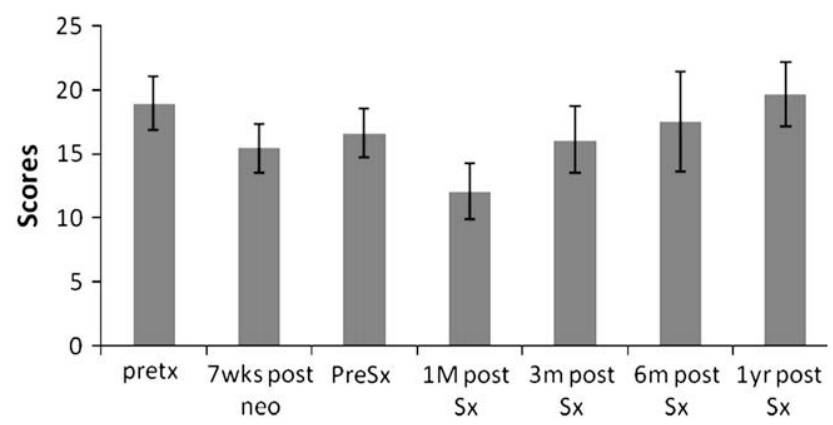

FIGURE 6. Functional well-being scores. Pretx, Before treatment; neo, 7 weeks after initiation of neoadjuvant therapy; presx, before resection; posts $x$, after resection.

Whether the difference in chemotherapy accounts for the difference in findings is open to question but supports the need for assessment of QOL in future clinical trials.

In analyzing the FACT-E subscales, it is an interesting observation that the physical and functional well-being scales decrease after neoadjuvant chemoradiation and again after surgical intervention, whereas the emotional and social well-being scales remain stable. This points to the resilience of the patient's perception of social and emotional support, despite physical and functional debility.

The EORTC-QLQ 30 and OES 18 have been used for much of the research in assessing HRQOL in patients with esophageal cancer in Europe. The EORTC-QLQ 30 has good correlation with the FACT, except in the social function role. The FACT social well- being domain focuses on sociability and social support, whereas the QLQ 30 social role reflects social activity limitations. The OES 18 and Esophageal Cancer subscale also have good correlations, especially with respect to eating and swallowing. ${ }^{11-16}$

The operative approach might influence HRQOL, at least in the early postoperative period. de Boer and coworkers ${ }^{17}$ reported decreased QOL scores 3 months after transthoracic esophagectomy compared with those after transhiatal esophagectomy, but these differences resolved subsequently. ${ }^{17}$ In our series a variety of surgical approaches were used,

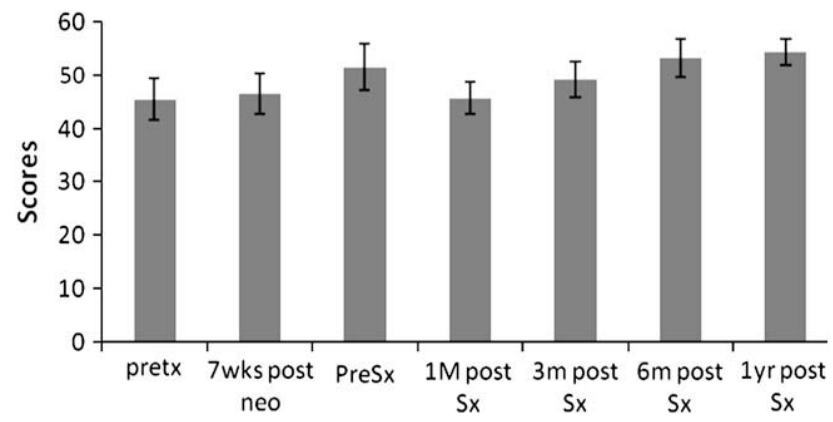

FIGURE 7. Esophageal subscale scores. Pretx, Before treatment; neo, 7 weeks after initiation of neoadjuvant therapy; presx, before resection; postsx, after resection. 
whereas details of the surgical approach or postoperative complications are not provided in the reports by Blazeby and colleagues or Brooks and associates.

Postoperative complications, including anastomotic leak, cardiopulmonary complications, and operative technical complications, have been reported to affect HRQOL at 6 months' follow-up. ${ }^{18} \mathrm{We}$ found no significant correlation between complications and HRQOL scores at 6 months compared with baseline scores when we examined the effect of anastomotic leak, pneumonia, respiratory failure, and postoperative sepsis (data not shown). However, this might be related to the small sample size of our study.

Significant but transient adverse effects on HRQOL related to neoadjuvant chemoradiotherapy were reported by Bottomley and associates, ${ }^{19}$ but as found in our study, scores returned to baseline levels before surgical intervention. However, others have reported that patients who had preoperative chemoradiotherapy did not achieve their baseline QOL score, even at 9 months. ${ }^{16}$ Reynolds and colleagues ${ }^{20}$ reported overall HRQOL scores did not return to baseline levels after either surgical intervention alone or neoadjuvant chemoradiation followed by surgical intervention until 6 months postoperatively, and even at 12 months there were significantly reduced scores in terms of physical and functional roles, but there was no difference between the groups. When we examined the component subscales of the FACT in our patients, we found that the physical and functional well-being scores were less than baseline scores at 6 months, but the functional well-being score was back to baseline by 12 months, whereas the physical well-being score lagged slightly. Nevertheless, combining all component subscales, the overall QOL scores were greater than baseline values.

In a randomized study of chemoradiation versus chemoradiation plus surgical intervention, HRQOL scores were significantly worse at the first follow-up visit (3 months) in the surgical patients, but this difference resolved by the second visit at 6 months, and over time, HRQOL scores remained similar in both arms. ${ }^{21}$ This supports our finding that the effect of neoadjuvant chemoradiation on HRQOL score is transient, and this strategy should be pursued in an effort to improve survival.

Several groups have suggested the baseline HRQOL scores are predictive of survival. Blazeby and colleagues ${ }^{14}$ reported that baseline HRQOL scores were not associated with postoperative morbidity but were significantly associated with survival at 6 months postoperatively after adjusting for known risk factors. A similar finding has been reported for patients treated with primary radiotherapy, and in particular, physical function role at baseline was predictive of survival. ${ }^{21,22}$ We did not find an association between baseline HRQOL score and early postoperative survival $(P=.80)$; however, our sample size is small, and there were only 3 deaths in the first 6 months. We did, however, find that increasing FACT-E scores postoperatively over time were predictive of improved survival $(P=0.04)$.
Combined modality therapies should continue to be explored in an effort to improve survival in this devastating disease. HRQOL should be assessed in addition to pathologic response rates and survival. Measurement of HRQOL provides investigators with an added dimension with which to compare different treatment regimens in a disease in which improvements in survival are modest and treatment morbidity and toxicities are considerable.

We thank Pfizer for generously supported this study by providing irinotecan for the study patients.

\section{References}

1. Darling G, Eton D, Sulman J, Casson A, Cella D. Validation of the functional assessment of cancer therapy esophageal cancer subscale. Cancer. 2006;107: 854-63.

2. Lerut T, Nafteux P, Moons J, et al. Three-field lymphadenectomy for carcinoma of the esophagus and gastroesophageal junction in 174 R0 resections: impact on staging, disease-free survival, and outcome: a plea for adaptation of TNM classification in upper-half esophageal carcinoma. Ann Surg. 2004;240:962-72.

3. Alexiou C, Khan OA, Black E, et al. Survival after esophageal resection for carcinoma: the importance of the histologic cell type. Ann Thorac Surg. 2006;82: 1073-7.

4. Fok M, Wong J. Cancer of the oesophagus and gastric cardia. Standard oesophagectomy and anastomotic technique. Ann Chir Gynaecol. 1995;84:179-83.

5. Visbal AL, Allen MS, Miller DL, Deschamps C, Trastek VF, Pairolero PC. Ivor Lewis esophagectomy for esophageal cancer. Ann Thorac Surg. 2001;71:1803-8.

6. Swanson SJ, Batirel HF, Jalitsch MT, et al. Transthoracic esophagectomy with radical mediastinal and abdominal lymphnode dissection and cervical esophagogastrostomy for esophageal carcinoma. Ann Thorac Surg. 2001;72:1918-24.

7. Swisher SG, Holmes EC, Hunt KK, et al. The role of neoadjuvant therapy in surgically resectable esophageal cancer. Arch Surg. 1996;131:819-24.

8. Walsh TN, Noonan N, Hollywood D, Kelly A, Keeling N, Hennessy PJ. A comparison of multimodal therapy and surgery for esophageal adenocarcinoma. N Engl J Med. 1996;33:462-7.

9. Urschel JD, Vasan H, Blewett CJ. A meta analysis of randomized controlled trials that compared neoadjuvant chemotherapy and surgery to surgery alone for resectable esophageal cancer. Am J Surg. 2002;183:274-9.

10. Malaiserie SC, Untch B, Aranha GV. Neoadjuvant chemoradiation for locally advanced esophageal cancer: experience at a single institution. Arch Surg. 2004;139:532-8.

11. Tamim WZ, Davidson RS, Quinlan RM, et al. Neoadjuvant chemotherapy for esophageal cancer: is it worthwhile? Arch Surg. 1998;133:722-6.

12. Knox J, Darling G, Guindi M, et al. A phase II study to assess the efficacy of combined preoperative irinotican I/cisplatin (C) chemotherapy and conformal radiotherapy (RT) followed by surgery for potentially resectable esophageal cancer. J Clin Oncol. 2004;22(14S):4063.

13. Blazeby JM, Conroy T, Hammerlid F, et al. Clinical and psychometric validation of an EORTC questionnaire module, the EORTC QLQ-OES18, to assess quality of life of patients with oesophageal cancer. Eur J Cancer. 2003;39: 1384-94.

14. Blazeby JM, Farndon JR, Donovan J, Alderson D. A prospective longitudinal study examining the quality of life of patients with esophageal carcinoma. Cancer. 2000;88:1781-7.

15. Viklund P, Wengstrom Y, Rouvelas I, Lindblad M, Lagergren J. Quality of life and persisting symptoms after oesophageal cancer surgery. Eur J Cancer. 2006; 42:1407-14.

16. Brooks JA, Kesler KA, Johnson CS, Ciatta D, Brown JW. Prospective analysis of quality of life after surgical resection for esophageal cancer: preliminary results. J Surg Oncol. 2002;81:185-94.

17. de Boer AG, van Lanschot JJ, van Sandick JW, et al. Quality of life after transhiatal compared with extended transthoracic resection for adenocarcinoma of the esophagus. J Clin Oncol. 2004;22:4202-8.

18. Viklund P, Lindblad M, Lagergren J. Influence of surgery related factors on quality of life after esophageal or cardia cancer resection. World J Surg. 2005;29:841.

19. Bottomley A, Flechtner H, Efficace F, et al. Health related quality of life outcomes in cancer clinical trials. Eur J Cancer. 2005;41:1697-709. 
20. Reynolds JV, McLaughlin R, Moore J, Rowley S, Ravi N, Byrne PJ. Prospective evaluation of quality of life in patients with localized oesophageal cancer treated by multimodality therapy or surgery alone. Br J Surg. 2006; 93:1084-90.

21. Bonnetain F, Bouche $\mathrm{O}$, Michel $\mathrm{P}$, et al. A comparative longitudinal quality of life study using the Spitzer quality of life index in a randomized multicenter phase III trial (FFCD9102): chemoradiation followed by surgery compared with chemoradiation alone in locally advanced squamous resectable thoracic esophageal cancer. Ann Oncol. 2006; 17:827-34

22. Fang FM, Tsai WL, Chiu HC, Kuo WR, Hsiung CY. Quality of life as a survival predictor for esophageal squamous cell carcinoma treated with radiotherapy. Int J Radiat Oncol. 2004;58:1394-404.

\section{Discussion}

Dr Kenneth A. Kesler (Indianapolis, Ind). For many years, we have measured indicators, such as operative mortality, levels of morbidity, and cure rates, to determine the success of treatment for locally advanced esophageal cancer, and we have made reasonable progress with respect to all of these particular variables over time. The operative risks for these operations have become acceptable. Induction therapy with cisplatin-based chemotherapy and concurrent radiation therapy has, for the most part, become accepted as the treatment of choice for patients with adequate performance status. As the authors of this study point out, however, overall survival improvements with induction therapy have been unfortunately modest, and meaningful survival benefits are probably limited to the subset of patients who demonstrate a significant or complete pathologic response. Until we have some mechanism to identify the subset of patients who will significantly benefit from platin-based induction therapy or, better yet, we have more effective chemotherapy agents, many patients with locally advanced esophageal cancer will not only not derive survival benefit from induction therapy but, as this prospective study demonstrates, will experience morbidity, including a temporary loss of QOL, which is significant in patients with limited median survival.

We therefore acknowledge the efforts of Dr Darling and her colleagues in Toronto, who have taken the next step to measure HRQOL as an important outcome variable. This study will not only serve as a point of reference with respect to QOL outcomes for this particular induction treatment strategy but hopefully as a starting point at which QOL instruments are routinely included in future prospective clinical trials for locally advanced esophageal cancer.

I have 3 questions. In this study you used the FACT-E questionnaire to quantitate HRQOL, which is an instrument you have validated in a previous study. Do you believe, however, future refinements to this instrument might be helpful, such as refinements to better capture potentially serious side effects after esophagectomy, such as dumping, reflux, or delayed gastric emptying, which might not be identified with this instrument or any other currently used instruments for that matter?

Dr Darling. Thank you, Dr Kesler. I think that is a very good point.

The item generation for the esophageal cancer subscale was developed from patients with recently diagnosed esophageal cancer rather than from patients after esophagectomy. I agree that it probably does not completely address postesophagectomy problems. We have not initiated any refinements, but I think it warrants further study. I think that we are seeing a little bit of those problems when we look at the eating index after surgical intervention. As we who treat these patients all know, their eating can be significantly affected by dumping syndrome or other problems after esophagectomy, and I think that is being reflected in the eating index. However, it is probably worthwhile for us to take another look at it and to address some of those postesophagectomy issues.

Dr Kesler. Although the patients in this study returned to baseline HRQOL scores relatively quickly after both induction therapy and esophagectomy, the baseline score was measured after disease diagnosis. At diagnosis, most of these patients were symptomatic with dysphagia and weight loss, not to mention having experienced the psychologic consequences of being told they have a cancer that is not frequently cured. Do you think it is possible to somehow establish a baseline HRQOL score reflecting both physical and mental status before illness that would help differentiate the effect of the disease from the effect of the treatment and additionally provide a more accurate reference point to compare against QOL measurements over time?

Dr Darling. That is a very good question also. I think it would be difficult because most of these patients are referred already knowing the diagnosis. The fact that the emotional well-being score remains stable throughout the treatment protocol and thereafter speaks to the resilience of some of these patients with respect to that component of QOL. Therefore I do not think that it would be particularly different if we somehow picked them up before they actually had the diagnosis. We know that if we compare QOL with the Short Form-36 (SF-36), which is designed for normal persons, and measure QOL in patients with cancer, more specifically esophageal cancer, that their QOL is significantly less than we would see in healthy subjects, but I am not sure how to capture them before they have actually been given the diagnosis.

Dr Kesler. Lastly, the finding that patients who died within 1 year of diagnosis, presumably of recurrent disease, demonstrated a significant decrease in QOL compared with 1-year survivors seems fairly intuitive and would be expected. Did the decrease in QOL observed in these patients happen to precede any clinical or radiographic evidence of cancer? As a corollary, do you think this represents a potential marker that can be clinically useful to detect recurrence?

Dr Darling. The decrease in QOL scores did precede the radiologic diagnosis of recurrence. These patients were all on study, and therefore they were receiving routine computed tomographic scans looking for recurrence. But the QOL scores decreased and were not recovering before any imaging changes, and therefore we were already worried. I am sure we all have had those patients in our practice. They just do not get better after esophagectomy, and you are trying to figure out why. I think the FACT-E might be clinically useful in the future as a marker for recurrent disease. I have certainly already adopted it. When I have that patient who is just not getting better, I start looking harder. Just to be clear, these patients were not aware that they had recurrence, and we were not aware that they had recurrence at the time those questionnaires were completed. I do think it will be a useful marker.

Dr Kesler. Thank you. Congratulations.

Dr Darling: Thank you very much.

Dr Scott J. Swanson (New York, NY). I would like to follow up on that last point because it is very intriguing. Do you think if you had not 
operated on those patients that they would have had a steady decrease in their QOL scores? Is there something about the operation or the intensity of the treatment unmasking their ability to handle cancer, and is it actually speeding up dissemination in some way? What do you think is the actual mechanism that is going on there?

Dr Darling. We know historically that patients who were not cured by means of esophagectomy had a median survival of 9 to 12 months. I think we are actually just seeing that same time course in these patients for whom our treatment protocol did not improve their survival. I do not think it is the effect of the operation or the induction that causes that decrease in QOL score. I think we would have seen it anyway.

Dr Douglas E. Wood (Seattle, Wash). Gail, I am interested in whether you think that the scale that you used, a cancer-specific QOL scale, might be indeed what we want to capture, or might we actually be interested in a more general QOL scale, such as SF-36, for these patients? This is very important work. There is clearly a bias in our medical colleagues that patients have a poor QOL relating to treatment, including esophagectomy, for esophageal cancer. I do not know enough about the scale that you used to know the differences and the nuances and whether our argument might be stronger if we chose a generalized scale.
Dr Darling. Well, I think they are complementary. If you want to compare a healthy population with a disease-specific population, a cancer population, you can use the SF-36. Therefore it depends on your question. We are particularly interested in comparing cancer therapies. We are trying to cure more esophageal cancer but at a price of the toxicity. Therefore our goal in using a cancer-specific instrument was to use it in the future for comparing different treatment protocols. The SF-36 would be useful in addressing a different question, such as whether patients with esophagectomy get back to a "normal" QOL score.

Dr Alec Patterson (St Louis, Mo). Gail, I enjoyed the article.

I noticed that $20 \%$ of the patients did not complete the program.

Dr Darling. Yes.

Dr Patterson. Were they all from Toronto? I mean, did you have any handle on the quality of the induction therapy?

Dr Darling. They were essentially all from Toronto. Some of the chemotherapy was delivered elsewhere, but in fact, all the patients who did not complete it were actually at our center. Some were patients who were not overly enthusiastic about chemotherapy and were not tolerant of any side effects. They did not stop for specific toxicity reasons. It was not a case of an outside oncologist not supporting the trial. 